\title{
Auf dem Weg zur ganzen Gesundheit
}

Dieser Jahrgang unserer Zeitschrift FORSCHENDE KOMPLEMENTÄRMEDIZIN UND KLASSISCHE NATURHEILKUNDE markiert ihr 10-jähriges Jubiläum. Sie ist damit unter den vier international verbreiteten Zeitschriften, die sich mit Komplementärmedizin und unkonventionellen medizinischen Richtungen befassen, die älteste. Nicht nur das unterscheidet sie von anderen: Sie ist eine Zeitschrift mit dezidiert europäischem Schwerpunkt, die international wahrgenommen wird. Dies wird durch einen steigenden Impact-Faktor dokumentiert (bei aller Fragwürdigkeit dieses bibliometrischen Instrumentes). Aber auch folgende kleine Anekdote mag das belegen: Kürzlich fragte mich Richard Hammerschlag, ein internationaler Fachmann der Akupunkturforschung, auf einer Tagung des «Samueli Institute for Information Biology» nach ein paar Details einer Arbeit aus unserer Zeitschrift, die sich mit unkonventioneller Diagnostik von Akupunkturpunkten und ihrer Reliabilität beschäftigte, und liess sich ein paar Stellen, die er nicht verstand, von mir übersetzen. Diese Arbeit aus dem Jahr 1998 [1] hat, wie manch andere auch, die in dieser Zeitschrift publiziert wurde, Marksteine gesetzt, die hoffentlich auch zukünftig der europäischen Forschung auf diesem Gebiet Gehör verschaffen werden.

Und FORSCHENDE KOMPLEMENTÄRMEDIZIN UND KLASSISCHE NATURHEILKUNDE ist die einzige Zeitschrift, in der in nennenswertem Umfang konzeptuelle Arbeiten publiziert werden. Damit meine ich solche, die nicht nur empirische Daten über Wirksamkeit und Unwirksamkeit unkonventioneller Behandlung oder Brauchbarkeit unkonventioneller Diagnostik zusammentragen, sondern die auch versuchen, eine Brücke zu schlagen zur konventionellen Medizin der Universitäten. Ich vermeide absichtlich den von Hahnemann geprägten Kampfbegriff der «Schulmedizin», weil ich der Meinung bin, dass es höchste Zeit ist, das Denken in Dichotomien und die Sprache der verfeindeten Lager aufzugeben, deren Auseinandersetzung nur mit Sieg der einen und Niederlage der anderen Gruppe enden kann. Vielmehr zeigt sowohl die Forschungspraxis als auch die Versorgungsnot, dass nur ein ganzheitlicher Ansatz weiterhilft, bei dem über einen Dialog der beiden Medizinkulturen ein gemeinsames Ziel erreichbar wird: die ganze Gesundheit nicht nur für ein paar Begüterte und Gebildete, die den Sinn einer komplementärmedizinischen Behandlung verstanden haben und sie sich leisten können. Nein, sondern die ganze Gesundheit für möglichst viele.

Die «ganze Gesundheit» meint Gesundheit in einem ganzheitlichen Sinn sowie Gesundheit für die Gesamtheit der Bevölkerung. Diese Denkfigur ist bei uns in Deutschland seit den rassenhygienischen Ergüssen nationalsozialistisch gleichgeschalteter Mediziner verpönt. Dennoch wird es Zeit, dass wir eine Sprache für diese soziale Dimension der Gesundheit finden, die dieses Tabu überwindet. Denn sonst wird uns die Leere der öffentlichen Kassen diese Sprache notgedrungen lehren.

Dieses Thema der «Gesundheit», und ich möchte ergänzen der «ganzen Gesundheit», haben wir zum Jubiläumsthema dieses 10. Jahrgangs unserer Zeitschrift erkoren. Wir sind stolz darauf, mit Klaus Michael Meyer-Abich einen der führenden Köpfe der deutschen Naturphilosophie in unserer Schriftleitung zu haben. Er hat schon in der Vergangenheit immer wieder das Thema vertieft und neue Dimensionen aufgezeigt [2-6]. Er eröffnet den Reigen der eingeladenen Beiträge [7]. Diese sind, anders als die gewöhnlich publizierten, nicht begutachtet worden. Vielmehr haben wir renommierte Autoren eingeladen, rund um den Begriff «Gesundheit» Aspekte aufzuzeigen und herauszugreifen, die diesen aus einer ganzheitlichen Sicht beleuchten oder Arbeiten zusammenfassen, die uns einen Schritt näher an ein solches Verständnis bringen. Mindestens eine eingeladene Arbeit wird im kommenden Jahr in jedem Heft zu finden sein. Prof. Meyer-Abich beginnt die Serie mit einer philosophischen Reflexion darüber, was es heisst «gesund» oder «ganz» zu sein, zu werden oder zu bleiben. Ich will ihm nicht vorgreifen. Aber soviel an vorbereitender und begleitender Analyse sei mir gestattet:

Es hat mich schon immer fasziniert, dass es eine indogermanische Sprachwurzel gibt, die Wurzel «hal», aus der die Begriffe «heil», «heilig» im Deutschen, «whole», «holy», «hallow» im 
Englischen, und durch Lautverschiebungen abgeleitet vermutlich auch die Begriffe «hagios - heilig» bzw. «hygieia - Gesundheit» im Griechischen herkommen. Diese sprachliche Verwandtschaft lehrt uns, dass im ursprünglichen Sprachverständnis die Gesundheit im Sinne der körperlichen Unversehrtheit, das «Heilsein» nur als Ganzsein, und dieses wiederum nur als «Heiligsein» begreifbar sind. Damit ist nicht das «Bravsein» gemeint, das viele noch aus einem fehlgeleiteten Religionsunterricht mit diesem Wort verbinden, sondern das Eingeordnetsein in das grössere Ganze der Lebensabläufe dieser Welt, die damit gleichzeitig als etwas begriffen und geehrt werden, das jenseits unserer kleinen, kontingenten und beschränkten individuellen Existenz waltet und das einzelne Leben, so brüchig und gescheitert es sein mag, birgt. «Heilig» meint aber nicht nur das passive Eingebundensein und das mehr oder weniger grummelnde Hinnehmen des Weltverlaufs. Es hat auch die Konnotation des aktiven Mittuns. Dieser ganze Lebensablauf, das sind wir, jeder einzelne, mit seinen Handlungen und Unterlassungen, mit seinen konstruktiven und destruktiven Gewohnheiten, Gedanken und Intentionen. Gesundheit entsteht dort, wo wir, uns einordnend in diesen Weltverlauf, versuchen ganzheitlich - «heilig» - zu sein, nicht nur im Hinblick auf unser körperliches Wohlbefinden, sondern auch in unseren sozialen Beziehungen, in unseren Handlungen und in unserem Denken. Das Ganze der Welt im Blick kann jede kleine Handlung, die Art und Weise der Entsorgung des Abfalls oder der Genuss der Nahrung, die Art unseres Fortkommens im Verkehr oder in der Berufslaufbahn, einen Beitrag zu unserer Ganzwerdung und damit Gesunderhaltung leisten, oder eben nicht. Und dies gilt nicht nur für uns als Einzelne, sondern auch für uns alle als sozialer Organismus, der die gegenseitigen Pflichten und Hilfen im Sozialsystem regelt. Dass es damit nicht zum Besten steht, lehrt uns ein Blick in die Zeitungen, wenn wir es nicht schon am eige- nen Leibe erfahren haben. Auch hier sind neue Denkansätze gefragt, neue Konzepte, die von grundlegend anderen Voraussetzungen ausgehen. Meyer-Abich bietet ein solches Konzept in seinem Beitrag an, das auf Vorstellungen Victor von Weizsäckers, des grossen Psychosomatikers, zurückgeht. Dieses Konzept sieht die individuelle Gesundheit viel stärker, als wir das gewohnt sind, aus der Perspektive der sozialen und naturgemässen Bindungen oder Bindungslosigkeit, und macht Gesundheitspolitik nicht zu einer Notwehrstrategie, sondern zu einer langfristig planenden Präventivarbeit. Es bleibt nur zu hoffen, dass mindestens der eine oder andere im Sozial- und Gesundheitsbereich verantwortlich Tätige diese Gedanken aufgreift und in konkrete politische Konzepte umsetzt.

Diesen ganzheitlichen Begriff von Gesundheit also wollen wir in diesem Jubiläumsjahrgang betrachten. Wir werden ihn manchmal philosophisch, wie in diesem Heft, manchmal stärker aus interdisziplinär medizinischer Sicht, manchmal mit spekulativen theoretischen Konzepten und manchmal aus spiritueller Perspektive beleuchten. Immer aber wird es uns darum gehen, einen kleinen Beitrag zum Verständnis dessen zu leisten, worum die Komplementärmedizin kreist: die ganze Gesundheit, die Gesundheit als ganzheitlich verstandener Begriff, die Gesundheit des Ganzen. Denn dies ist die tiefste Einsicht, deren sich auch die Medizin nicht verschliessen kann und darf: Gesundheit des einzelnen Teiles kann es nicht geben ohne die Gesundheit des Ganzen, und Gesundheit des Ganzen erzeugt Gesundheit der einzelnen Teile. Dies gilt für den individuellen Organismus und seine Organe und Organbausteine. Dies gilt für das einzelne Individuum und seine nächste soziale Bezugsgruppe, die Familie. Dies gilt für den Einzelnen und seine Einbettung in die soziale Gruppe. Und dies gilt gleichermassen für uns alle als Teile eines Weltganzen. Es ist heilsam, sich daran von Zeit zu Zeit zu erinnern.

H. Walach, Freiburg i. Br.

\section{Literatur}

1 Treugut H, Görner C, Lüdtke R, Burghardt V: Reliabilität der energetischen Meridianmessung mit Prognos A $^{\circledR}$. Forsch Komplementärmed 1998;5: 284-289.

2 Meyer-Abich KM: Soziopsychosomatik in «Forschende Komplementärmedizin und Klassische Naturheilkunde»? (Editorial). Forsch Komplementärmed Klass Naturheilkd 2002;9:5-6.
3 Meyer-Abich KM: Wie naturwissenschaftlich ist die Medizin? - Das Leitbild Gesundheit in der Medizin und in den Umweltwissenschaften. Forsch Komplementärmed Klass Naturheilkd 2001;8:34-38.

4 Meyer-Abich KM: Die nichtnaturwissenschaftliche Zeit des Lebens und der Krankheit. Forsch Komplementärmed Klass Naturheilkd 2000;7:154-158.

5 Meyer-Abich KM: Kann sogar die Prävention falsch sein? - Über das militärische Denken in der Medizin (Editorial). Forsch Komplementärmed Klass Naturheilkd 2000;7:177-178.
6 Meyer-Abich KM: Naturheilkunde oder Komplementärmedizin? (Editorial). Forsch Komplementärmed 1999;5:244-246.

7 Meyer-Abich KM: Wie ganzheitlich ist die Gesundheit? - Von Hippokrates und Platon bis zu einer künftigen sozio-psychosomatischen Situationstherapie. Forsch Komplementärmed Klass Naturheilkd 2003;10:35-39. 